\title{
$\beta$-Sheet Core of Tau Paired Helical Filaments Revealed by Solid-State NMR
}

Venita Daebel, ${ }^{\perp}$ Subashchandrabose Chinnathambi, ${ }^{\nabla, \#}$ Jacek Biernat, ${ }^{\nabla, \#}$ Martin Schwalbe, ${ }^{\perp}$ Birgit Habenstein, ${ }^{\perp}$ Antoine Loquet, ${ }^{\perp}$ Elias Akoury, ${ }^{\perp}$ Katharina Tepper, ${ }^{\nabla, \#}$ Henrik Müller ${ }^{\perp, \dagger}$ Marc Baldus, ${ }^{\perp, \ddagger}$ Christian Griesinger, ${ }^{\perp}$ Markus Zweckstetter, ${ }^{\perp, \otimes}$ Eckhard Mandelkow, ${ }^{*, \nabla, \#}$ Vinesh Vijayan, ${ }^{*, \perp, \S}$ and Adam Lange*, ${ }^{*}$

${ }^{\perp}$ NMR-based Structural Biology, Max Planck Institute for Biophysical Chemistry, Am Fassberg 11, 37077 Göttingen, Germany

${ }^{\nabla}$ DZNE, German Center for Neurodegenerative Diseases, Ludwig-Erhard-Allee 2, 53175 Bonn, Germany

${ }^{\#}$ CAESAR Research Center, Ludwig-Erhard-Allee 2, 53175 Bonn, Germany

${ }^{\otimes}$ DZNE, German Center for Neurodegenerative Diseases, Grisebachstraße 5, 37077 Göttingen, Germany

Supporting Information

ABSTRACT: One of the hallmarks of Alzheimer's disease is the self-assembly of the microtubule-associated protein tau into fibers termed "paired helical filaments" (PHFs). However, the structural basis of PHF assembly at atomic detail is largely unknown. Here, we applied solid-state nuclear magnetic resonance (ssNMR) spectroscopy to investigate in vitro assembled PHFs from a truncated three-repeat tau isoform (K19) that represents the core of PHFs. We found that the
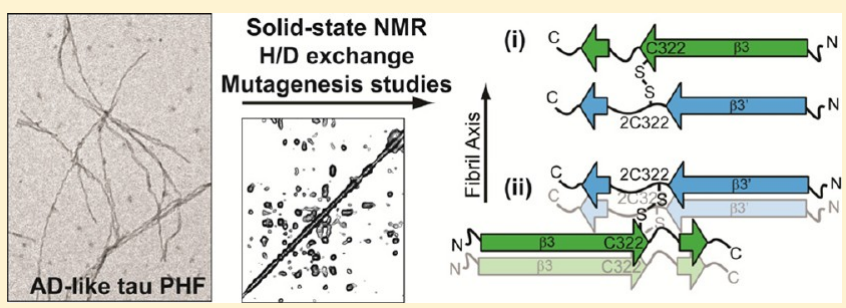
rigid core of the fibrils is formed by amino acids V306 to S324, only 18 out of 99 residues, and comprises three $\beta$-strands connected by two short kinks. The first $\beta$-strand is formed by the wellstudied hexapeptide motif VQIVYK that is known to self-aggregate in a steric zipper arrangement. Results on mixed $\left[{ }^{15} \mathrm{~N}:{ }^{13} \mathrm{C}\right]$ labeled K19 fibrils show that $\beta$-strands are stacked in a parallel, in-register manner. Disulfide bridges formed between C322 residues of different molecules lead to a disturbance of the $\beta$-sheet structure, and polymorphism in ssNMR spectra is observed. In particular, residues K321-S324 exhibit two sets of resonances. Experiments on K19 C322A PHFs further confirm the influence of disulfide bond formation on the core structure. Our structural data are supported by H/D exchange NMR measurements on K19 as well as a truncated four-repeat isoform of tau (K18). Site-directed mutagenesis studies show that single-point mutations within the three different $\beta$-strands result in a significant loss of PHF aggregation efficiency, highlighting the importance of the $\beta$ structure-rich regions for tau aggregation.

\section{INTRODUCTION}

The microtubule-associated protein $\operatorname{tau}^{1}$ is expressed in the adult human brain mainly in six different isoforms. Due to alternative splicing, two N-terminal inserts (N1, N2; Figure 1) and the second out of four repeats (R2) in the C-terminal microtubule-binding domain can be present or absent. ${ }^{2,3}$ The major physiological role of tau is to bind to and stabilize microtubules facilitating axonal transport. However, upon phosphorylation, tau detaches from microtubules and selfassembles into amyloid fibrils. ${ }^{4}$ In Alzheimer's disease (AD) and other tauopathies, hyperphosphorylated tau aggregates into paired helical filaments (PHFs), 5 which represent the major constituents of neurofibrillary tangles (NFTs). ${ }^{6}$ A progressive accumulation of NFTs is one of the neuropathological hallmarks of $\mathrm{AD}^{7}$ Hence, a structural model of tau PHFs could be helpful in the development of small compounds that prevent tau aggregation., 8

PHFs can be structurally divided into two regions: (i) a rigid amyloid core and (ii) a highly flexible fuzzy coat of more than 200 residues that transiently attaches to the amyloid core. ${ }^{10}$
Protease digestion and solvent accessibility studies indicated that the core of PHFs is mainly built from amino acids belonging to the repeats $\mathrm{R} 2$ (present only in four-repeat isoforms) and R3. ${ }^{11,12}$ Furthermore, circular dichroism (CD) spectropolarimetry and Fourier transform infrared (FTIR) spectroscopy, together with X-ray diffraction and selected area electron diffraction, have indicated the presence of cross- $\beta$ structure, where $\beta$-strands run roughly perpendicular to the fiber axis. ${ }^{13-15}$ In addition, electron paramagnetic resonance (EPR) studies have suggested that, within tau fibrils, $\beta$-strands are arranged in-register and parallel. ${ }^{16,17}$

Despite these improvements in understanding the principles of tau fibril formation, a detailed insight into the residue-specific structural arrangement of $\beta$-strands formed by the amino acids in R2 and R3 is still not available. In this regard, magic-angle spinning (MAS) solid-state nuclear magnetic resonance (ssNMR) spectroscopy constitutes a powerful method to

Received: February 3, 2012

Published: August 3, 2012 


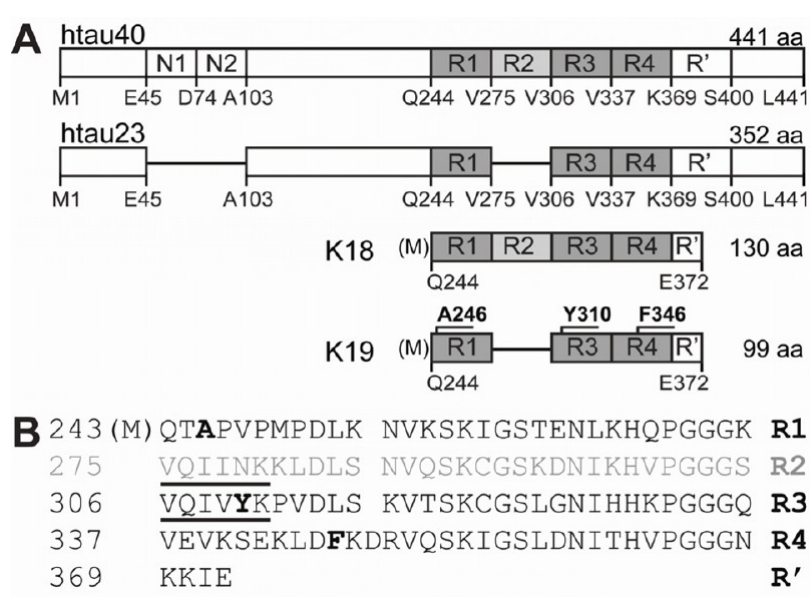

Figure 1. Tau isoforms and constructs K18 and K19. (A) Tau is expressed in the adult human brain in six isoforms with htau 40 as the longest and htau 23 as the shortest one. While K18 is a truncated form of htau40, K19 originates from htau23. Repeat domains common to all constructs are shown in dark gray, whereas the alternatively spliced R2 is depicted in light gray. The highlighted amino acids (aa) in K19 are unique within the construct sequence and are located in different repeats. (B) Amino acid sequence of the constructs K19 and K18. Amino acids from R2 (K18) are shown in light gray. Underlined residues belong to the hexapeptide motifs that are known to promote fibril formation. Amino acids in bold letters are exclusively present in one of the repeats and have unique chemical shifts that make them easily identifiable in NMR spectra.

obtain structural information on insoluble and noncrystalline biological systems ${ }^{18-20}$ including amyloid fibrils. ${ }^{21-34}$

Here, we employed MAS ssNMR to investigate PHFs assembled in vitro from the 99 amino acid (aa) tau construct K19 (Figure 1). It corresponds to the juvenile htau23 isoform of tau, containing a three-repeat version of the microtubulebinding domain comprising R1, R3, and R4, but lacking the $\mathrm{N}$ and C-terminal domains. ${ }^{35}$ A previous study on K19 PHFs using ssNMR has shown that these fibrils consist of a rigid core surrounded by regions of higher flexibility. The most rigid, water-inaccessible part of the fibrils was found to be formed by repeat R3, whereas R1 and R4 are more water-accessible. ${ }^{36}$ However, in this study a pronounced structural heterogeneity resulting in substantial line broadening hampered the determination of the exact arrangement of $\beta$-structure within the core. Based on an improved sample preparation and an extensive labeling strategy, we present here a detailed ssNMR analysis of the K19 core structure. Additionally, we conducted $\mathrm{H} / \mathrm{D}$ exchange NMR experiments to probe the accessibility of the core residues in a site-specific manner. To assess whether the identified core residues of K19 are also relevant in longer tau constructs, we also studied PHFs formed by K18, a fourrepeat construct (comprising R2; Figure 1), by H/D exchange. Further insight into PHF assembly was obtained by investigating the aggregation behavior of $\mathrm{K} 19$ variants mutated at specific sites in the identified core region of the fibrils.

\section{RESULTS AND DISCUSSION}

Flexible Regions of Tau K19 PHFs in R1 and R4. In order to detect the flexible segments of K19 PHFs which do not belong to the rigid core, we performed INEPT-based CCTOBSY ssNMR experiments. ${ }^{37-39}$ In the resulting spectra only resonances of residues with increased mobility (on the ps to ns time scale) are observed.

The K19 sequence features three unique amino acids, A246 in R1, Y310 in R3, and F346 in R4 (Figure S1A), with distinct chemical shifts that can be easily identified. While the INEPTCC-TOBSY spectra exhibit resonances with unambiguous random coil chemical shifts of alanine (Figure S1B) and phenylalanine (Figure S1C, black spectrum), tyrosine resonances are clearly absent (Figure S1C, dashed circles). To unequivocally validate that the signal in Figure S1C originates from phenylalanine, we repeated the experiment on the K19 ${ }_{\text {KFLVrev }}$ sample (for a detailed description of the different samples see SI). Since phenylalanine is not $\left[{ }^{13} \mathrm{C},{ }^{15} \mathrm{~N}\right]$-labeled, the corresponding peaks vanish in the spectrum.

The appearance of F346 and A246 as well as the absence of Y310 in the INEPT-CC-TOBSY spectra shows that R1 and R4 are much more flexible than R3. Furthermore, the absence of the Y310 signals implies that the resonances in the spectra do not originate from soluble, monomeric K19 protein. In this case, the INEPT-CC-TOBSY spectra would contain signals from all residues of the molecule.

ssNMR Studies on K19 PHFs Identify a Well-Defined Rigid Fibril Core. ${ }^{13} \mathrm{C}-{ }^{13} \mathrm{C}$ correlations for residues of the rigid fibril core were obtained using PDSD experiments ${ }^{40}$ with mixing times of 20,150, and $500 \mathrm{~ms}$ to gain intraresidue, sequential, and medium- and long-range correlations, respectively (see, e.g., Figure S2A). Other experiments were performed (e.g., DREAM; see Figure S3), but the maximum number of spin systems could be observed in the PDSD experiments. Sequential assignment was also obtained by means of NCACX and NCOCX experiments. ${ }^{41}$

As expected from the previous study, ${ }^{36}$ the measured resonances cover only a subset of the 99 aa of the entire K19 sequence. With V306-S324 (see Table S1), in total 18 residues are assigned to the rigid K19 PHF core. These residues belong to the repeat $\mathrm{R} 3$, which corroborates the results from the INEPT-based experiments.

To probe the missing $\sim 80 \%$ of the K19 sequence, we conducted PDSD experiments $\left(t_{\text {mix }}=20 \mathrm{~ms}\right)$ at variable temperatures of $-4,5$, and $15{ }^{\circ} \mathrm{C}$ (see Figures 3A, S4, and S5). While the spectra recorded at 5 and $15^{\circ} \mathrm{C}$ resemble each other in peak intensity and resolution, spectra recorded at $-4{ }^{\circ} \mathrm{C}$ exhibit significantly increased signal intensity and broader lines as well as a large number of additional peaks.

Thus, when aggregated into PHFs, $\sim 80 \%$ of the K19 residues are highly dynamic and become sufficiently rigid to be observed in dipolar-based ssNMR experiments only when the sample is frozen.

This result is reminiscent of observations by Jaroniec and coworkers who found that signals from approximately 100 residues (i.e., $\sim 80 \%$ of all residues) of human $\operatorname{PrP}(23-144)$ amyloid fibrils are not detected above approximately $-20{ }^{\circ} \mathrm{C}$ in dipolar-based ssNMR experiments. ${ }^{42}$

Spectra of K19 PHFs Reveal Two Conformations for Residues K321-S324. Surprisingly, in PDSD spectra of a sample, where only cysteine, tyrosine, and leucine are $\left[{ }^{13} \mathrm{C},{ }^{15} \mathrm{~N}\right]$-labeled (Figure S2B), we unambiguously detected two cysteine resonances, despite the fact that only one, $\mathrm{C} 322$, is present in the K19 sequence. Both resonances (in the following: C322 and 2C322) exhibit chemical shifts typical for oxidized cysteines involved in disulfide bond (DSB) formation. ${ }^{43,44}$ 


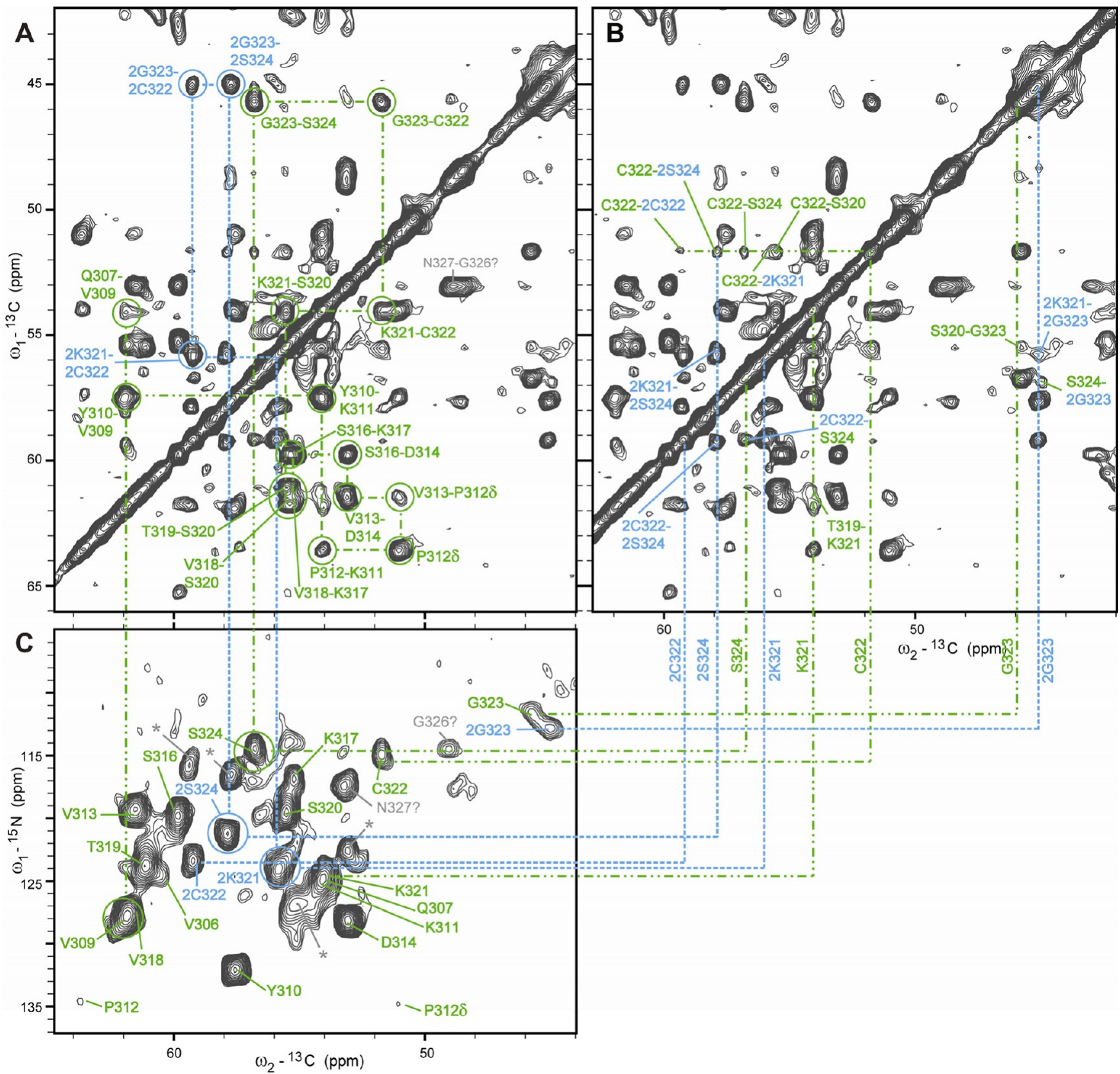

Figure 2. Sequential resonance assignment. (A,B) $2 \mathrm{D}\left[{ }^{13} \mathrm{C},{ }^{13} \mathrm{C}\right]$-PDSD spectrum of $\left[2-{ }^{13} \mathrm{C}\right]$-glycerol-labeled $\mathrm{K} 19$ PHFs $\left(\mathrm{K} 19_{2 \mathrm{glyc}} t_{\mathrm{mix}}=500 \mathrm{~ms}\right)$ recorded on an $850 \mathrm{MHz}$ spectrometer at $11 \mathrm{kHz}$ MAS at $11^{\circ} \mathrm{C}$. Sequential correlations of resonance sets 1 (green) and 2 (blue) (A), and mediumrange as well as interset correlations (B) are indicated. (C) $2 \mathrm{D}\left[{ }^{15} \mathrm{~N},{ }^{13} \mathrm{C}\right]-\mathrm{NCA}$ spectrum of $\mathrm{K} 19_{\text {2glyc }}$ acquired on an $800 \mathrm{MHz}$ spectrometer at 11 $\mathrm{kHz}$ MAS and $5{ }^{\circ} \mathrm{C}$. Note that Leu $\mathrm{C} \alpha$ is not labeled in the $\mathrm{K} 19_{\text {gglyc }}$ sample. Correlations labeled in gray could not be assigned unambiguously.

The presence of two conformationally different cysteines in the fibril core could be confirmed by PDSD experiments on a sparsely labeled K19 ${ }_{2 \text { glyc }}$ sample (Figure 2), where we observed two sets of resonances for residues K321-S324 (set 1, K321S324 shown in green; set 2, 2K321-2S324 shown in blue). Notably, not only sequential and medium-range connections within the sets (Figure 2A) were observed, but five long-range correlations between the two sets as well (C322-2K321, C322-2C322, C322-2S324, 2C322-S324, S324-2G323; Figure $2 \mathrm{~B}$ ). Furthermore, both sets are sequentially connected to the preceding residues-that are observed only onceindicating a structural difference only in the C-terminal part of the PHF core.
To unequivocally ensure that the splitting of residues K321S324 is due to the presence of an intermolecular DSB, we mutated C322 to alanine. The NCA spectrum of K19 C322A PHFs clearly reveals a simplified resonance pattern compared to that of wild-type (WT) K19 (Figure 3B) with two interesting findings: (i) Instead of two cysteine resonances, one strong alanine signal is observed, with chemical shifts indicative of $\beta$ sheet structure. Also residues $\mathrm{K} 321-\mathrm{S} 324$ do not give rise to two sets of signals with equal intensity any more. Nevertheless, peak doubling still occurs to a small extent, as seen, e.g., by a second weak alanine signal (2A322 in Figure 3B). (ii) In the WT K19 spectra, some peaks appear that do not show sequential correlations and could therefore not be assigned (marked with asterisks in Figures 2C and 3B). These 

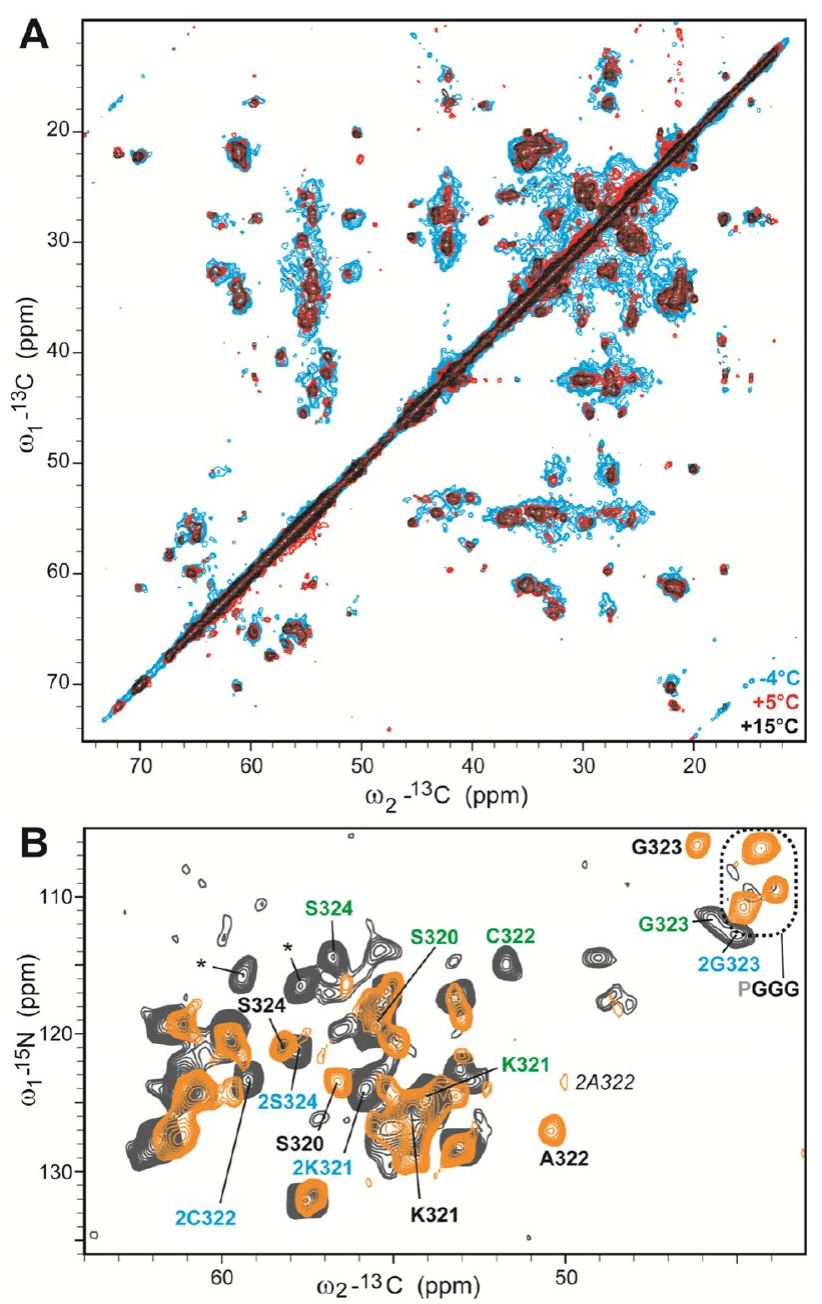

Figure 3. Probing of temperature effects and disulfide bond elimination. (A) Overlay of PDSD spectra $\left(t_{\text {mix }}=20 \mathrm{~ms}\right)$ of K19C322A PHFs recorded on an $800 \mathrm{MHz}$ spectrometer at $11 \mathrm{kHz}$ MAS at different temperatures (blue, $-4{ }^{\circ} \mathrm{C}$; red, $5{ }^{\circ} \mathrm{C}$; black, $15{ }^{\circ} \mathrm{C}$ ). (B) Comparison of $2 \mathrm{D}\left[{ }^{15} \mathrm{~N},{ }^{13} \mathrm{C}\right]-\mathrm{NCA}$ spectra of K19 C322A PHFs (orange) and K19 WT PHFs (dark gray; same K19 $2_{2 \mathrm{glyc}}$ spectrum as in Figure 2C), both recorded on an $800 \mathrm{MHz}$ spectrometer at $11 \mathrm{kHz}$ MAS and $5{ }^{\circ} \mathrm{C}$. Resonance assignments of sets 1 (green) and 2 (blue) are based on $\mathrm{K} 19_{2 \text { glyc }}$ while black assignment labels are for the mutant. For the sake of clarity, only assignments for S320-S324 are shown. Residues in the dashed box belong to glycines of a PGGG motif. The correlations marked with asterisks could not be assigned in K19 WT and occur only weakly in the mutant in the noise.

resonances may arise from additional peak doubling due to multiple conformations in the K19 WT. In contrast, they are absent or occur only weakly in spectra of K19 C322A PHFs. The observed spectral simplification suggests that the study of C322A K19 PHFs could be a promising approach for future ssNMR studies that aim at structure determination of tau fibrils. However, in this study we focused on the WT K19 construct. In future studies we will analyze in detail chemical shift changes between the two forms and perform experiments on C322A K19 PHFs that yield long-range distance information.

Secondary Structure Analysis of the K19 PHF Core Residues. In Figure 4A,B, secondary chemical shifts (SCSs)indicative of the secondary structure ${ }^{45}$ - for residues V306S320 (gray) as well as the two sets K321-S324 and 2K3212S324 are depicted. Based on our chemical shift assignment,

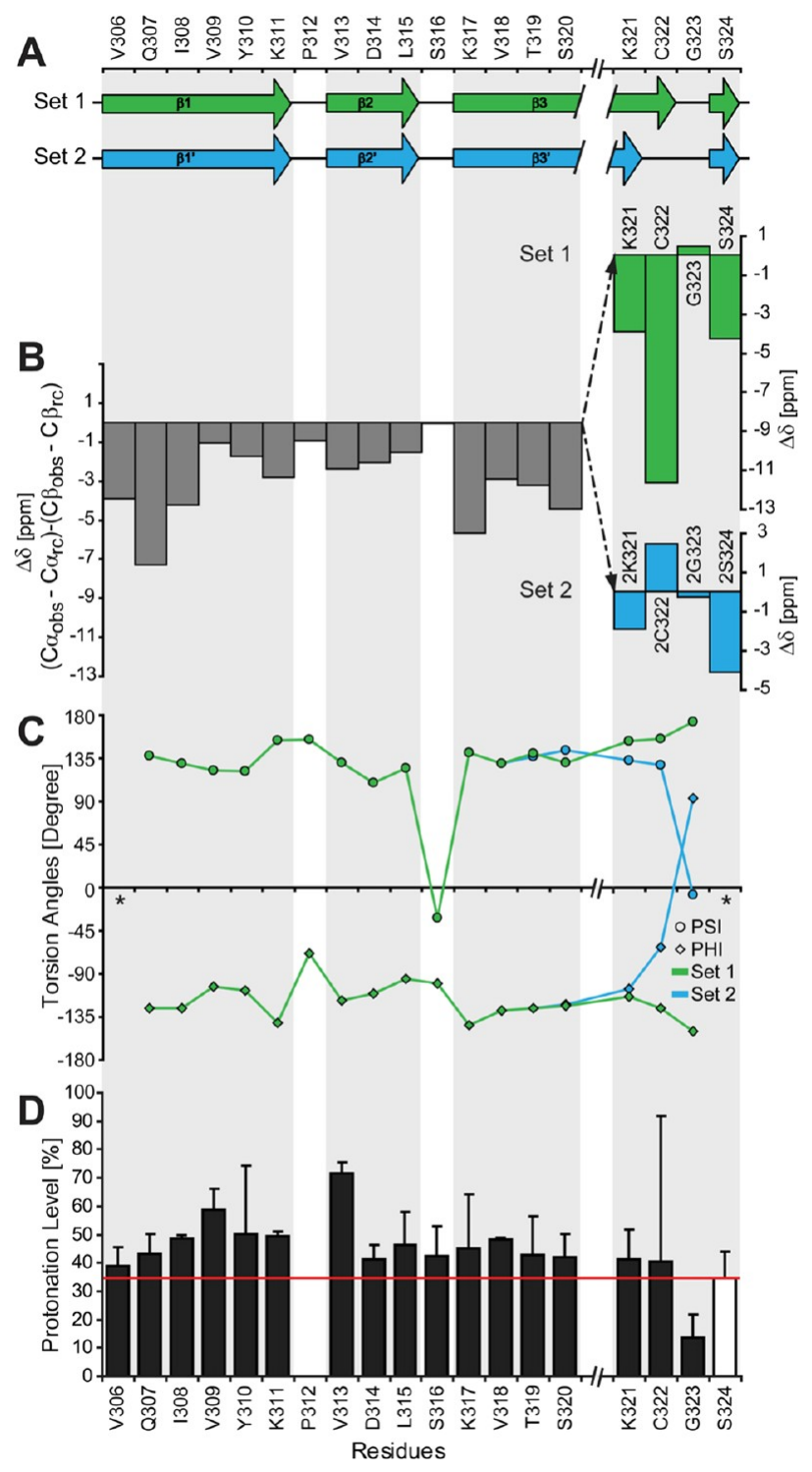

Figure 4. Secondary chemical shift (SCS) analysis of K19 PHFs. (A) Cartoon representation of the secondary structure of the two sets (set 1 , green; set 2, blue). (B) Consecutive negative values indicate a $\beta$ strand conformation; discontinuities in the negative values indicate a kink between $\beta$-strands. The SCS analysis reveals the presence of three $\beta$-strands interrupted by kinks at P312, a known $\beta$-strand breaker, and S316. The first $\beta$-strand is formed by the hexapeptide motif. From $\mathrm{K} 321$ to $\mathrm{S} 324$ the sequence is split into two sets of resonances. (C) A TALOS+ analysis, predicting $\phi(\diamond)$ and $\psi(O)$ dihedral torsion angles for sets 1 (green) and 2 (blue), corroborates the results from the SCS analysis. (All predictions are classified by TALOS+ as "good", except for 2G323.) (D) Protonation levels of the assigned rigid core of K19 filaments after $36 \mathrm{~h}$ forward-exchange to $\mathrm{D}_{2} \mathrm{O}$ as a function of residue number. The horizontal red line indicates the average protonation of all $\mathrm{K} 19$ residues. The open bar is an averaged value from overlapped residues (see also Figure S6).

and in line with the previous ssNMR study, ${ }^{36}$ the fibril core is predominantly found in $\beta$-strand conformation. This is further corroborated by backbone dihedral angle predictions obtained from the program TALOS ${ }^{46}$ (Figure $4 \mathrm{C}$; predictions for all residues were classified as "good", except for 2G323).

Exceptions occur for residues P312, S316, and G323, as well as $2 \mathrm{C} 322$ and $2 \mathrm{G} 323$. The SCSs of these residues indicate the presence of kinks that disrupt the $\beta$-strands (Figure 4A) and 
might change the characteristic inside-outside order of side chains observed in a fully extended conformation.

Previous studies have shown that the hexapeptide ${ }^{306}$ VQI$\mathrm{VYK}^{311}$ in $\mathrm{R} 3$ displays $\beta$-sheet propensity in disordered fulllength tau and is furthermore able to self-assemble into fibrils by itself. ${ }^{9,47-49}$ Consistently, SCSs identify the first $\beta$-strand from V306-K311. At P312 a kink is probably formed, because proline is a known $\beta$-strand breaker and the $\mathrm{C} \alpha$ chemical shift of P312 is larger than the random coil value, indicating a non- $\beta$ strand conformation. P312 is followed by a second, short $\beta$ strand, V313-L315, and another kink located at residue S316. In molecules belonging to set 1 , the third strand extends from $\mathrm{K} 317$ to $\mathrm{C} 322$, followed by a further kink at G323. In set 2 molecules, the third $\beta$-strand comprises only residues $\mathrm{K} 317-$ $2 \mathrm{~K} 321$, and a larger kink is formed by $2 \mathrm{C} 322$ and $2 \mathrm{G} 323$.

Previously it was shown that a spin label attached to C322 in PHF tau can be quenched by a reducing agent, indicating that the side chain of $\mathrm{C} 322$ is not fully buried in the fibrillar core $^{10}$ - in line with formation of a kink at C322.

Downstream of this kink, S324 as well as 2S324 chemical shifts indicate $\beta$-strand structure. However, as there is no unambiguous assignment for the consecutive residues, it is not clear whether a fourth $\beta$-strand follows.

While deletion of the C-terminus of full-length tau tends to enhance fibril formation, the deletion of residues D314-S320 abrogates fibrillation, ${ }^{50}$ probably as it eliminates strands $\beta 2$ and $\beta 3$. Interestingly, ${ }^{314} \mathrm{DLSK}^{317}$ shows conformational propensity for a turn in natively unfolded tau in solution as well as in chemical denaturant, as observed by SCS and residual dipolar coupling analysis. ${ }^{48,51}$ This indicates that the turn conformation might be a precursor to fibril conformation similar to the hexapeptide motif.

H/D Exchange Experiments Reveal a Protective Environment in the Identified Core Region. To probe the solvent accessibility of the residues forming the core of PHFs, we conducted H/D exchange experiments, using solution-state $\mathrm{NMR}^{52}$ As the fibrillar state is not accessible by solution-state NMR, solvent-protected amide protons were identified as described previously (for a detailed description see SI). In short, after an initial forward exchange of PHFs to $99.9 \%$ $\mathrm{D}_{2} \mathrm{O}$, the PHFs are dissolved in a strongly denaturing buffer with $50 \% \mathrm{D}_{2} \mathrm{O} / 50 \% \mathrm{H}_{2} \mathrm{O}$. Thus, the $\mathrm{H} / \mathrm{D}$ exchange rates can be determined by solution-state NMR, and with these rates the residual protonation after forward exchange is back-calculated. Solvent-protected sites will display a higher residual protonation relative to solvent-exposed sites.

In agreement with the ssNMR results, the most protected region in K19 PHFs belongs to residues I308-V313. with a certain degree of protection visible for residues D314-K321 as well (Figure 4D). In contrast, the two other repeats, R1 and R4, are more solvent-exposed, with the majority of residues displaying protonation levels below or equal to the average value (Figure S6). A short, consecutive stretch in R1 from D252-K257 also displays above average protonation levels similar to D314-K321, suggesting some degree of protection for this region. However, this is not apparent for the same region in K18 (see below). Residues D252-K257 showed a lower intensity relative to other residues, in particular in R3, and, as the error bars indicate, the above average protonation may be the result of a poor fit.

Furthermore, we probed using PHFs formed by K18 how repeat R2 contributes to the fibril core (Figures S6-S8). The $\mathrm{H} / \mathrm{D}$ exchange behavior is similar for K19 and K18 (Figure S7), and the most protected region in both $\mathrm{K} 19$ and $\mathrm{K} 18$ is repeat R3 (Figures S6 and S8). The protective environment provided by the fibril core in R3 might explain why residue $\mathrm{Y} 310$ is the only tyrosine in tau that is nearly resistant to chemical modification by site-specific nitration ${ }^{53}$ and that gives the most extensive shift changes in fluorescence spectra even in fulllength tau during PHF aggregation. ${ }^{11} \mathrm{~K} 18$ residues of repeat R2 are also protected from exchange with solvent (Figures S6 and S8). In particular, the hexapeptide ${ }^{275} \mathrm{VQIINK}^{280}$ at the $\mathrm{N}$ terminus of $\mathrm{R} 2$ is highly protected, indicating its involvement in the rigid fibril core of K18 PHFs.

Aggregation Behavior of K19 Single Mutants Supports the NMR-Derived Structural Data. The $\beta$-strand $\beta 1$ of the PHF core corresponds to the hexapeptide ${ }^{306} \mathrm{VQIVYK}^{311}$ that has been studied extensively. ${ }^{14,47,49,54}$ It was shown that $\beta$ strand-breaking proline point mutations within this motif, e.g., I308P, abrogate PHF formation. ${ }^{14}$ On the other hand, the influence of strands $\beta 2$ and $\beta 3$ (corresponding to V313-C322/ V313-2K321) on fibril formation and stability is largely unknown. Hence, in the current study we introduced singlepoint mutations in this part of the K19 core and followed the aggregation behavior of the mutant proteins. The kinetics of aggregation was monitored fluorimetrically using the thioflavin $\mathrm{S}(\mathrm{ThS})$ assay (Figure $5 \mathrm{~A}-\mathrm{C}$ ). The presence and morphology of PHFs were monitored by electron microscopy (EM, Figure 5D).

First, K19 proline mutants were designed, which were expected to be inhibitory for aggregation by disrupting the $\beta$ strands $\beta 1, \beta 2$, and $\beta 3$, respectively (Figure $5 \mathrm{~A}, \mathrm{D}$ ). As expected, the mutations $\mathrm{V} 313 \mathrm{P}$ and $\mathrm{T} 319 \mathrm{P}$ disrupt the $\beta$-strands and prevent fibril formation. The most pronounced inhibition was observed for V313P, which leads to nearly complete abrogation of aggregation, comparable to the previously described I308P mutation, furthermore, we generated the mutations L315E within $\beta 2$ and $S 320 \mathrm{E}$ as part of $\beta 3$, but also L $325 \mathrm{E}$, which is just outside of the assigned region (Figure $5 \mathrm{~B}$ ). These mutations disrupt PHF formation to different degrees. The farthest $\mathrm{C}$ terminal mutation, L325E, shows the least drastic effect.

With S320V, a hydrophilic serine is replaced by a hydrophobic valine. Figure 5C illustrates the aggregation kinetics of S320V, which is even faster than of the WT.

Figure 5D summarizes the structures observed by negative stain EM. The control sample of K19 WT PHFs shows abundant long filaments, predominantly with twisted substructure. The morphology of K19 S320V fibrils is indistinguishable from PHFs of K19 WT. In the remaining micrographs we can distinguish between two different morphologies: (i) fragmented short filaments formed by the mutants S320E and L325E and (ii) amorphous deposits without filaments for the mutants I308P, V313P, L315E, and T319P.

In summary, the aggregation behavior of the proline mutants provides evidence that the $\beta$-structure is highly important for fibril formation in the overall region of residues 306-325.

A hydrophobic influence on the stability of the PHF core was probed by mutating hydrophobic to charged residues. These mutations abrogate WT-like fibril formation. In contrast, replacing S320 by a more hydrophobic valine actually leads to an enhancement of fibrillation, indicating hydrophobic interactions to be a substantial stabilizing factor for K19 PHFs.

Intermolecular Stacking of Tau K19 PHFs Is Parallel and In-Register. To elucidate the intermolecular arrangement of tau K19 molecules to form the cross- $\beta$ structure, a two- 


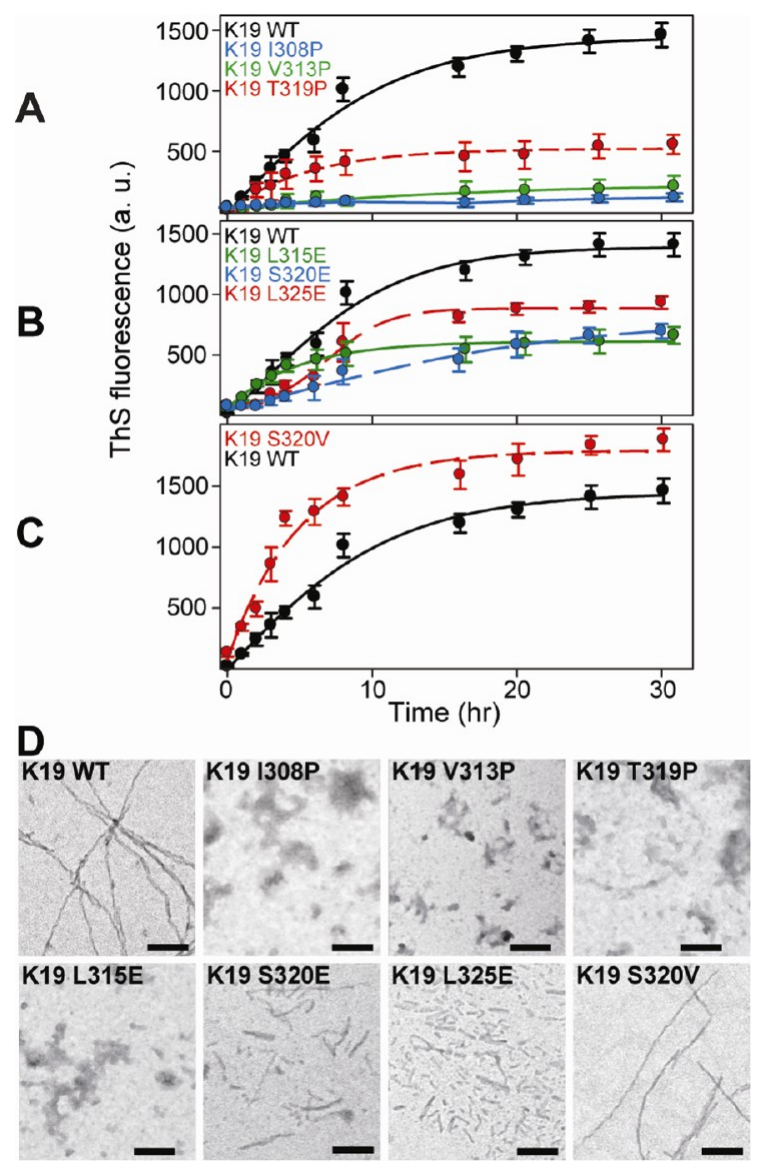

Figure 5. Site-directed mutagenesis studies on K19 PHFs. (A-C) PHF assembly of different K19 constructs monitored by ThS fluorescence: (A) K19 WT and $\beta$-strand-breaking proline mutants I308P, V313P, and T319P; (B) K19 inhibitory mutants L315E, S320E, and L325E; and (C) K19 stimulatory mutant S320V. (D) Electron micrographs of the different K19 mutants (in the presence of heparin; scale bars $=200 \mathrm{~nm}$ ).

dimensional ${ }^{15} \mathrm{~N}-{ }^{13} \mathrm{C}$ PAIN-CP ${ }^{55}$ spectrum of a mixed labeled K19 $1: 1$ sample was measured (Figure 6, blue). These PHFs were aggregated from an equimolar mixture of $\left[{ }^{15} \mathrm{~N}\right]$-labeled and $\left[{ }^{13} \mathrm{C}\right]$-labeled $\mathrm{K} 19$ protein. Since no ${ }^{15} \mathrm{~N}-{ }^{13} \mathrm{C}$-spin pairs are present within one K19 molecule of such mixed-labeled fibrils, only intermolecular ${ }^{15} \mathrm{~N}(\mathrm{i})-{ }^{13} \mathrm{C} \alpha(\mathrm{i})$ correlations are obtained in the PAIN-CP spectrum ${ }^{56}$ (Figure 6B). The spectrum overlays well with an NCA spectrum of the K19 ${ }_{\text {uni }}$ sample (Figure 6A, black) revealing a parallel, in-register arrangement of the $\beta$-strands along the fibril axis, in line with previous EPR studies. ${ }^{16,17}$

Notably, no intermolecular ${ }^{15} \mathrm{~N}(\mathrm{i})-{ }^{13} \mathrm{C} \alpha(\mathrm{i})$ correlations are detected for residues $\mathrm{K} 321-\mathrm{S} 324$ (and 2K321-2S324; orange circles in Figure 6A), indicating either that this part is not stacked parallel and in-register or that set 1 and set 2 molecules are stacked in an alternating arrangement (see also discussion in the next paragraph).

Intermolecular Disulfide Bonds Stabilize K19 PHFs. All investigated samples were prepared under conditions in which DSB formation can occur. The chemical shifts of the two assigned cysteines (C322 and 2C322) show that this happens indeed. Importantly, five long-range restraints correlating the two sets were detected in the PDSD $500 \mathrm{~ms}$ spectrum of the K19 2glyc $_{\text {sample. }}$

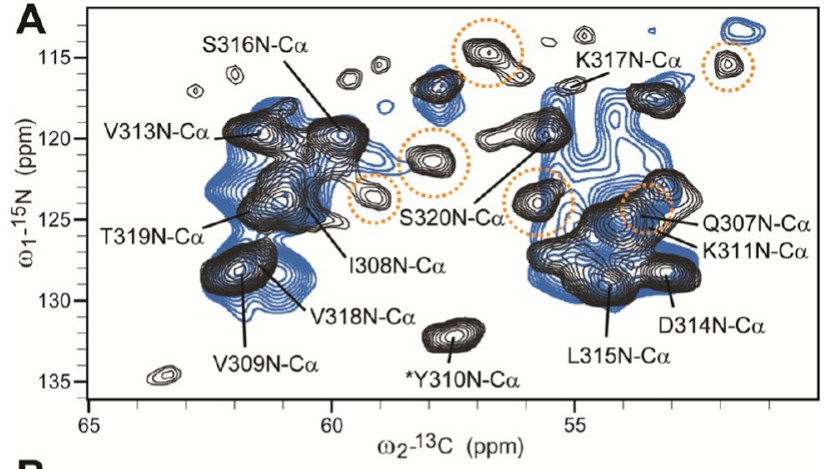

B

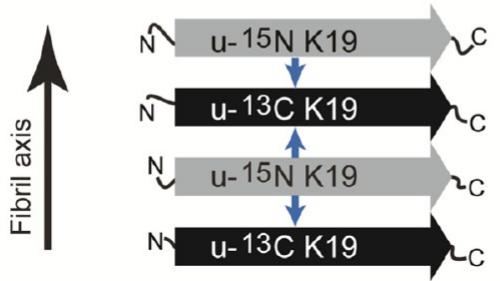

Figure 6. Intermolecular stacking of tau K19 PHFs. (A) Overlay of a $2 \mathrm{D}\left[{ }^{15} \mathrm{~N},{ }^{13} \mathrm{C}\right]$-PAIN-CP correlation spectrum (blue, $t_{\text {mix }}=5 \mathrm{~ms}$ ) of PHFs containing a $1: 1$ mixture of $\mathrm{u}-\left[{ }^{13} \mathrm{C}\right]$ - and $\mathrm{u}-\left[{ }^{15} \mathrm{~N}\right]$-labeled $\mathrm{K} 19$ molecules $\left(\mathrm{K} 19_{1: 1}\right)$ acquired on an $850 \mathrm{MHz}$ spectrometer at $18 \mathrm{kHz}$ MAS at $5{ }^{\circ} \mathrm{C}$ with a $2 \mathrm{D}\left[{ }^{15} \mathrm{~N},{ }^{13} \mathrm{C}\right]$-NCA spectrum (black) of $\mathrm{K} 19_{\text {uni }}$ recorded on an $850 \mathrm{MHz}$ spectrometer at $11 \mathrm{kHz}$ MAS at $7{ }^{\circ} \mathrm{C}$. The labeled resonances indicate that the $\beta$-strands are arranged in-register and parallel. Note that all intramolecular resonances in the NCA spectrum belonging to the doubled region (K321-S324/2K3212S324) do not appear in the PAIN-CP spectrum (orange circles). *The $\mathrm{Y} 310 \mathrm{~N}-\mathrm{C} \alpha$ correlation appears in the noise. (B) Intermolecular magnetization transfer is responsible for the correlations seen in the 2D PAIN-CP spectrum.

Because cysteine is a unique amino acid within the K19 sequence, the DSB must be formed intermolecularly (Figure 7). In principle, two possibilities are conceivable: (i) an intermolecular DSB within one protofilament along the fibril axis (Figure 7A), which is possible because of the parallel, inregister arrangement, ${ }^{57,58}$ or (ii) an inter-protofilament DSB or rather a DSB formed by symmetrically nonequivalent monomers in a lateral dimer within one protofilament (Figure 7B). ${ }^{59,60}$

Aggregation assays on K19 molecules under reducing conditions using DTT investigated by ThS and EM reveal that K19 is still able to form PHFs, but at a much slower rate. ${ }^{61}$ This strongly supports the notion that K19 intermolecular DSBs are an important factor for a fast and stable aggregation behavior, not least because DSB-linked tau dimers are known to successfully seed PHF aggregation. ${ }^{35}$

\section{CONCLUSION}

As tau aggregation into PHFs is one of the hallmarks of $\mathrm{AD}$, it is important to understand structural features of the fibril core as well as the fibrillation process itself. Our site-specific ssNMR study on K19 PHFs reveals a well-defined rigid core (V306S324; from R3 only) composed of three $\beta$-strands. Our data unambiguously disclose a parallel, in-register supramolecular arrangement, including the presence of intermolecular disulfide bonds between the tau monomer units.

Furthermore, our results support that the PHF core region is essential not only in K19 but in longer constructs, such as K18, as well. Ongoing ssNMR studies on the K19 C322A mutant 

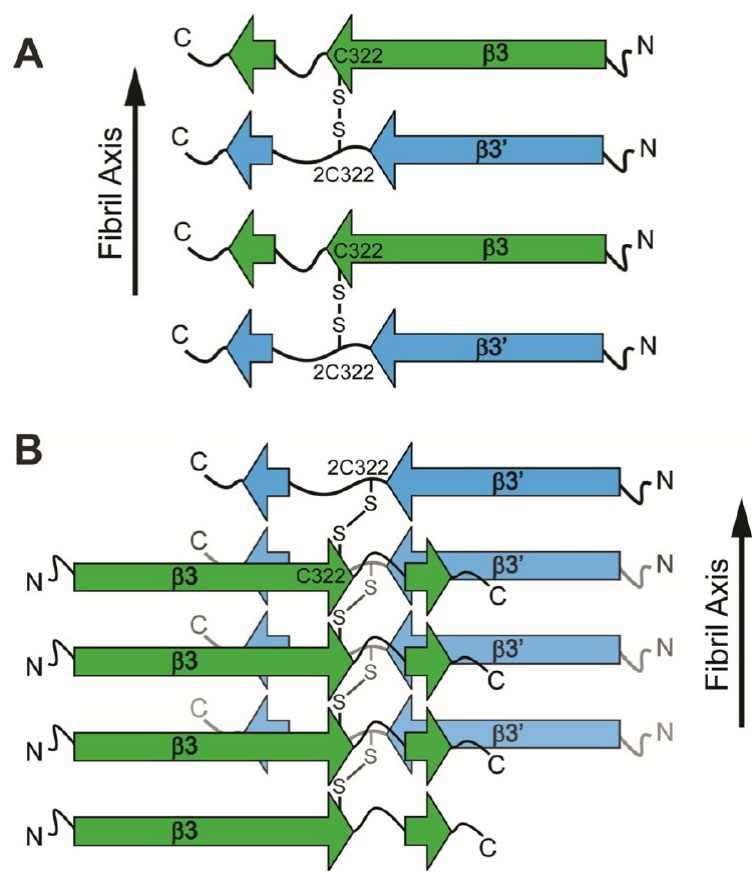

Figure 7. Models for cysteine disulfide bond formation within K19 PHFs. The molecules shown in green and blue relate to the two observed resonance sets for the residues close to C322. (A) DSB formation along the fibril axis within one protofilament. (B) Laterally formed DSB.

will be helpful to obtain unambiguous long-range distance restraints required for a high-resolution model of tau PHFs.

\section{MATERIALS AND METHODS}

WT and mutant K19 proteins were prepared ${ }^{62}$ and aggregation of tau was performed as described previously. ${ }^{36}$ ssNMR experiments were conducted on aggregated tau K19 samples (protein quantities of $\sim 10-$ $15 \mathrm{mg}$ ) using 4 or $3.2 \mathrm{~mm}$ triple-resonance $\left({ }^{1} \mathrm{H},{ }^{13} \mathrm{C},{ }^{15} \mathrm{~N}\right)$ MAS probes at static magnetic fields of 20,18.8, and 14.1 T (Bruker Biospin, Karlsruhe, Germany). Further details are given in the SI.

\section{ASSOCIATED CONTENT}

\section{S Supporting Information}

Detailed materials and methods; supplemental figures regarding assignment strategy and $\mathrm{H} / \mathrm{D}$ exchange data; chemical shift assignment table. This material is available free of charge via the Internet at http://pubs.acs.org.

\section{AUTHOR INFORMATION}

\section{Corresponding Author}

adla@nmr.mpibpc.mpg.de; vinesh@iisertvm.ac.in; mandelkow@dzne.de

\section{Present Addresses}

${ }^{\dagger}$ Forschungszentrum Jülich $\mathrm{GmbH}, 52425$ Jülich, Germany ${ }^{\ddagger}$ Utrecht University, Padualaan 8, $3584 \mathrm{CH}$ Utrecht, Netherlands

${ }^{\S}$ IISER Thiruvananthapuram, India

Notes

The authors declare no competing financial interest.

\section{ACKNOWLEDGMENTS}

We are grateful to Dr. E.-M. Mandelkow for discussions and I. Lindner and B. Angerstein for excellent technical assistance. We thank the Max Planck Society, the DFG (DFG ZW 71/7-1 and
Heisenberg scholarship DFG ZW 71/2-2 to M. Zweckstetter, Emmy Noether fellowship to A. Lange), and the European Molecular Biology Organization (long-term fellowship to A. Loquet) for financial support. V. Daebel was supported within the scope of the Graduiertenkolleg GRK 782 of the DFG.

\section{REFERENCES}

(1) Cleveland, D. W.; Hwo, S. Y.; Kirschner, M. W. J. Mol. Biol. 1977, 116, 227.

(2) Lee, G.; Cowan, N.; Kirschner, M. Science 1988, 239, 285.

(3) Goedert, M.; Spillantini, M. G.; Jakes, R.; Rutherford, D.; Crowther, R. A. Neuron 1989, 3, 519.

(4) Grundke-Iqbal, I.; Iqbal, K.; Tung, Y. C.; Quinlan, M.; Wisniewski, H. M.; Binder, L. I. Proc. Natl. Acad. Sci. U.S.A. 1986, 83, 4913.

(5) Iqbal, K.; del C. Alonso, A.; Chen, S.; Chohan, M. O.; El-Akkad, E.; Gong, C.-X.; Khatoon, S.; Li, B.; Liu, F.; Rahman, A.; Tanimukai, H.; Grundke-Iqbal, I. Biochim. Biophys. Acta, Mol. Basis Dis. 2005, 1739, 198.

(6) Lee, V. M. Y.; Balin, B. J.; Otvos, L., Jr.; Trojanowski, J. Q. Science 1991, 251, 675 .

(7) Lee, V. M. Y.; Goedert, M.; Trojanowski, J. Q. Annu. Rev. Neurosci. 2001, 24, 1121.

(8) Mandelkow, E.; von Bergen, M.; Biernat, J.; Mandelkow, E. M. Brain Pathol. 2007, 17, 83.

(9) Sievers, S. A.; Karanicolas, J.; Chang, H. W.; Zhao, A.; Jiang, L.; Zirafi, O.; Stevens, J. T.; Munch, J.; Baker, D.; Eisenberg, D. Nature 2011, 475, 96.

(10) Bibow, S.; Mukrasch, M. D.; Chinnathambi, S.; Biernat, J.; Griesinger, C.; Mandelkow, E.; Zweckstetter, M. Angew. Chem., Int. Ed. 2011, 50, 11520.

(11) Li, L.; von Bergen, M.; Mandelkow, E. M.; Mandelkow, E. J. Biol. Chem. 2002, 277, 41390.

(12) von Bergen, M.; Barghorn, S.; Muller, S. A.; Pickhardt, M.; Biernat, J.; Mandelkow, E. M.; Davies, P.; Aebi, U.; Mandelkow, E. Biochemistry 2006, 45, 6446.

(13) Berriman, J.; Serpell, L. C.; Oberg, K. A.; Fink, A. L.; Goedert, M.; Crowther, R. A. Proc. Natl. Acad. Sci. U.S.A. 2003, 100, 9034.

(14) von Bergen, M.; Barghorn, S.; Li, L.; Marx, A.; Biernat, J.; Mandelkow, E. M.; Mandelkow, E. J. Biol. Chem. 2001, 276, 48165.

(15) Giannetti, A. M.; Lindwall, G.; Chau, M. F.; Radeke, M. J.; Feinstein, S. C.; Kohlstaedt, L. A. Protein Sci. 2000, 9, 2427.

(16) Margittai, M.; Langen, R. Proc. Natl. Acad. Sci. U.S.A. 2004, 101, 10278.

(17) Margittai, M.; Langen, R. J. Biol. Chem. 2006, 281, 37820.

(18) McDermott, A. E. Curr. Opin. Struct. Biol. 2004, 14, 554.

(19) Tycko, R. Q. Rev. Biophys. 2006, 39, 1.

(20) Loquet, A. S., N.G.; Gupta, R.; Giller, K.; Riedel, D.; Goosmann, C.; Griesinger, C.; Kolbe, M.; Baker, D.; Becker, S.; Lange, A. Nature 2012, DOI: $10.1038 /$ nature 11079 .

(21) Parthasarathy, S.; Long, F.; Miller, Y.; Xiao, Y.; McElheny, D.; Thurber, K.; Ma, B.; Nussinov, R.; Ishii, Y. J. Am. Chem. Soc. 2011, $133,3390$.

(22) Nielsen, J. T.; Bjerring, M.; Jeppesen, M. D.; Pedersen, R. O.; Pedersen, J. M.; Hein, K. L.; Vosegaard, T.; Skrydstrup, T.; Otzen, D. E.; Nielsen, N. C. Angew. Chem., Int. Ed. 2009, 48, 2118.

(23) Jaroniec, C. P.; MacPhee, C. E.; Astrof, N. S.; Dobson, C. M.; Griffin, R. G. Proc. Natl. Acad. Sci. U.S.A. 2002, 99, 16748.

(24) Heise, H.; Hoyer, W.; Becker, S.; Andronesi, O. C.; Riedel, D.; Baldus, M. Proc. Natl. Acad. Sci. U.S.A. 2005, 102, 15871.

(25) Wasmer, C.; Lange, A.; Van Melckebeke, H.; Siemer, A. B.; Riek, R.; Meier, B. H. Science 2008, 319, 1523.

(26) Iwata, K.; Fujiwara, T.; Matsuki, Y.; Akutsu, H.; Takahashi, S.; Naiki, H.; Goto, Y. Proc. Natl. Acad. Sci. U.S.A. 2006, 103, 18119.

(27) Ferguson, N.; Becker, J.; Tidow, H.; Tremmel, S.; Sharpe, T. D.; Krause, G.; Flinders, J.; Petrovich, M.; Berriman, J.; Oschkinat, H.; Fersht, A. R. Proc. Natl. Acad. Sci. U.S.A. 2006, 103, 16248. 
(28) Vilar, M.; Chou, H. T.; Luhrs, T.; Maji, S. K.; Riek-Loher, D.; Verel, R.; Manning, G.; Stahlberg, H.; Riek, R. Proc. Natl. Acad. Sci. U.S.A. 2008, 105, 8637.

(29) Bertini, I.; Gonnelli, L.; Luchinat, C.; Mao, J.; Nesi, A. J. Am. Chem. Soc. 2011, 133, 16013.

(30) Loquet, A.; Giller, K.; Becker, S.; Lange, A. J. Am. Chem. Soc. 2010, 132, 15164.

(31) Petkova, A. T.; Yau, W. M.; Tycko, R. Biochemistry 2006, 45, 498.

(32) Helmus, J. J.; Surewicz, K.; Surewicz, W. K.; Jaroniec, C. P. J. Am. Chem. Soc. 2010, 132, 2393.

(33) Comellas, G.; Lemkau, L. R.; Nieuwkoop, A. J.; Kloepper, K. D.; Ladror, D. T.; Ebisu, R.; Woods, W. S.; Lipton, A. S.; George, J. M.; Rienstra, C. M. J. Mol. Biol. 2011, 411, 881.

(34) Kloepper, K. D.; Hartman, K. L.; Ladror, D. T.; Rienstra, C. M. J. Phys. Chem. B 2007, 111, 13353.

(35) Wille, H.; Drewes, G.; Biernat, J.; Mandelkow, E. M.; Mandelkow, E. J. Cell Biol. 1992, 118, 573.

(36) Andronesi, O. C.; von Bergen, M.; Biernat, J.; Seidel, K.; Griesinger, C.; Mandelkow, E.; Baldus, M. J. Am. Chem. Soc. 2008, 130, 5922.

(37) Burum, D. P.; Ernst, R. R. J. Magn. Reson. 1980, 39, 163.

(38) Andronesi, O. C.; Becker, S.; Seidel, K.; Heise, H.; Young, H. S.; Baldus, M. J. Am. Chem. Soc. 2005, 127, 12965.

(39) Baldus, M.; Meier, B. H. J. Magn. Reson., Ser. A 1996, 121, 65.

(40) Szeverenyi, N. M.; Sullivan, M. J.; Maciel, G. E. J. Magn. Reson.

1982, 47, 462.

(41) Baldus, M.; Petkova, A. T.; Herzfeld, J.; Griffin, R. G. Mol. Phys. 1998, 95, 1197.

(42) Helmus, J. J.; Surewicz, K.; Nadaud, P. S.; Surewicz, W. K.; Jaroniec, C. P. Proc. Natl. Acad. Sci. U.S .A. 2008, 105, 6284.

(43) Sharma, D.; Rajarathnam, K. J. Biomol. NMR 2000, 18, 165.

(44) Wang, Y.; Jardetzky, O. Protein Sci. 2002, 11, 852

(45) Luca, S.; Filippov, D. V.; van Boom, J. H.; Oschkinat, H.; de Groot, H. J. M.; Baldus, M. J. Biomol. NMR 2001, 20, 325.

(46) Shen, Y.; Delaglio, F.; Cornilescu, G.; Bax, A. J. Biomol. NMR 2009, 44, 213.

(47) Sawaya, M. R.; Sambashivan, S.; Nelson, R.; Ivanova, M. I.; Sievers, S. A.; Apostol, M. I.; Thompson, M. J.; Balbirnie, M.; Wiltzius, J. J. W.; McFarlane, H. T.; Madsen, A. O.; Riekel, C.; Eisenberg, D. Nature 2007, 447, 453.

(48) Mukrasch, M. D.; Biernat, J.; von Bergen, M.; Griesinger, C.; Mandelkow, E.; Zweckstetter, M. J. Biol. Chem. 2005, 280, 24978.

(49) von Bergen, M.; Friedhoff, P.; Biernat, J.; Heberle, J.; Mandelkow, E. M.; Mandelkow, E. Proc. Natl. Acad. Sci. U.S.A. 2000, 97, 5129.

(50) Abraha, A.; Ghoshal, N.; Gamblin, T.; Cryns, V.; Berry, R.; Kuret, J.; Binder, L. J. Cell. Sci. 2000, 113, 3737.

(51) Mukrasch, M. D.; Markwick, P.; Biernat, J.; Bergen, M.; Bernado, P.; Griesinger, C.; Mandelkow, E.; Zweckstetter, M.; Blackledge, M. J. Am. Chem. Soc. 2007, 129, 5235.

(52) Cho, M. K.; Kim, H. Y.; Fernandez, C. O.; Becker, S.; Zweckstetter, M. Protein Sci. 2011, 20, 387.

(53) Reynolds, M. R.; Berry, R. W.; Binder, L. I. Biochemistry 2005, 44, 1690.

(54) Goux, W. J.; Kopplin, L.; Nguyen, A. D.; Leak, K.; Rutkofsky, M.; Shanmuganandam, V. D.; Sharma, D.; Inouye, H.; Kirschner, D. A. J. Biol. Chem. 2004, 279, 26868.

(55) Lewandowski, J. R.; De Paepe, G.; Griffin, R. G. J. Am. Chem. Soc. 2007, 129, 728.

(56) Etzkorn, M.; Bockmann, A.; Lange, A.; Baldus, M. J. Am. Chem. Soc. 2004, 126, 14746.

(57) Almeida, A. M.; Li, R.; Gellman, S. H. J. Am. Chem. Soc. 2012, 134, 75 .

(58) Choi, J. H.; May, B. C.; Wille, H.; Cohen, F. E. Biophys. J. 2009, 97, 3187.

(59) Schmidt, M.; Sachse, C.; Richter, W.; Xu, C.; Fandrich, M.; Grigorieff, N. Proc. Natl. Acad. Sci. U.S.A. 2009, 106, 19813.
(60) Lopez Del Amo, J. M.; Schmidt, M.; Fink, U.; Dasari, M.; Fandrich, M.; Reif, B. Angew. Chem., Int. Ed. 2012, DOI: 10.1002/ anie.201200965.

(61) Barghorn, S.; Mandelkow, E. Biochemistry 2002, 41, 14885.

(62) Barghorn, S.; Biernat, J.; Mandelkow, E. Methods Mol. Biol. 2005, 299, 35. 